TRANSACTIONS OF THE

AMERICAN MATHEMATICAL SOCIETY

Volume 222, 1976

\title{
PRINCIPAL CO-FIBER BUNDLES
}

BY

\section{ELYAHU KATZ}

ABSTRACT. Principal co-fiber bundles are defined in the category of topological groups. They are Eckmann-Hilton duals of principal fiber bundles. A classification theorem is provided as well as an example which almost represents the most general case.

1. Introduction. The Eckmann-Hilton duality [4] works well as long as we are in the homotopy category. However in the category of topological spaces there are no duals to principal fiber bundles (p.f.b.), since any set with a comultiplication with a strict co-unit reduces to a point. This situation is avoided in [9] by turning to the category of simplicial groups. In this paper we define principal co-fiber bundles (p.c.b.) in the category of topological groups. We face very few surprises in the algebraic part of the problem since this part is similar to the study in [9]. The difficulties are in the topology.

The main result is the existence of two adjoint functors $\delta$ and $\epsilon$ which correspond p.f.b.'s to p.c.b.'s and under certain conditions are inverses to each other. These functors are applied to classify the p.c.b.'s. The proof of the classification theorem relies heavily on Milnor's work in [7]. We also present a typical example which in fact almost represents the most general case. This class of examples, up to homotopy equivalence, is the loops of a principal cofibration.

In $\S 3$ we point out a category of p.f.b.'s. $\$ 4$ contains the definition of a category of p.c.b.'s. In $\S \S 5,6$, and 7 , we define the two functors and describe the relations between them. The classification of p.c.b.'s is in $\S 8$. The example is in $\S 9$. Some proofs are in $\S 10$.

All spaces are pointed with base point $e$, which denotes also the identity of the groups. The disjoint union identifying base points is denoted by $V$.

I would like to thank Professor I. Berstein for suggesting the problem and for many discussions with regard to this problem.

Received by the editors December 20, 1974 and, in revised form, June 26, 1975.

AMS (MOS) subject classifications (1970). Primary 55F10; Secondary 22A05, 20E05, 20E30.

Key words and phrases. Principal fiber bundle, principal co-fiber bundle, co-group, free topological group, $k_{W}$ group. 
2. Preliminaries.

Definition A. [1] $F(X)$ is a free topological group with basis $(X, e)$, a pointed topological space, if $F(X)$ has the following properties: (i) $F(X)$ is a topological group with subspace $X$. (ii) $X-e$ generates $F(X)$ freely as a group. (iii) For any continuous map $\phi: X \rightarrow G$, where $G$ is a topological group, and $\phi(e)$ equals the identity element of $G$, then $\phi$ extends to a continuous homomorphism $\Phi: F(X) \rightarrow G$.

Definition B. (a) A $k_{W}$ space is a topological space $X$ with compact subsets $X_{n}$, such that: (i) $X=\bigcup_{n=1}^{\infty} X_{n}$. (ii) $X_{n+1} \supset X_{n}$ for all $n$. (iii) A subset $A$ of $X$ is closed if and only if $A \cap X_{n}$ is compact for all $n$. (b) By a $k_{w}$ decomposition, $X=\bigcup X_{n}$, we mean that $X_{n}$ have properties (i), (ii) and (iii). (c) $\mathrm{A} k_{W}$ group is a topological group which is also a $k_{W}$ space.

THEOREM A. (a) [1] For any completely regular space $X$, the group $F(X)$ exists. (b) [6] Let $X=\bigcup_{n=1}^{\infty} X_{n}$ be a $k_{W}$ decomposition of $X$. Then $F(X)$ exists and has the weak topology of $F_{n}(X)$. (c) [6] $F(X)$ also has a $k_{W}$ decomposition $F(X)=\bigcup_{n=1}^{\infty} F_{n}\left(X_{n}\right)$. The sets $F_{n}(X)$ and $F_{n}\left(X_{n}\right)$ are defined as follows: Let $X^{-1}$ be a copy of $X$ and let us denote $\widetilde{X}=X \vee X^{-1}$ the disjoint union of $X$ and $X^{-1}$, identifying the base points. $F_{n}(X)$ is the image of the projection of $(\widetilde{X})^{n}$ into $F(X)$, where the projection sends a word to its reduced form in $F(X) . F_{n}(X)$ is also given the induced topology of the projection described above. $F_{n}\left(X_{n}\right)$ is defined similarly.

Definition C. [2] Let $G_{1}$ and $G_{2}$ be two topological groups. Then, the topological group $G_{1} * G_{2}$ is said to be a free topological product of $G_{1}$ and $G_{2}$, if it has the following properties: (i) $G_{1}$ and $G_{2}$ are subgroups of $G_{1} * G_{2}$, with the subspace topology. (ii) The underlying group of $G_{1} * G_{2}$ is their free product as groups. (iii) If $\gamma_{1}$ and $\gamma_{2}$ are continuous homomorphisms of $G_{1}$ and $G_{2}$ into a topological group $H$, then there exists a continuous homomorphism $\Gamma: G_{1} * G_{2} \rightarrow H$ such that $\Gamma=\gamma_{i}$ on $G_{i}, i=1,2$.

TheOREM B. (a) [2] Let $G_{1}, G_{2}$ be topological groups, then $G_{1} * G_{2}$ exists. (b) [8] Let $G_{i}=\bigcup_{j} G_{i}^{j}$ be $k_{W}$ decompositions for the topological groups $G_{1}, G_{2}$. Then, $G_{1} * G_{2}=\bigcup_{j} h\left(\left(G_{1}^{j} \vee G_{2}^{j}\right)^{j}\right)$ is a $k_{W}$ decomposition where $h$ sends $a$ word to its reduced form in $G_{1} * G_{2}$.

Definition D. The pair $\langle G, \psi\rangle$ where $G$ is a topological group and $\psi$ a homomorphism $\psi: G \rightarrow G * G$ is a co-group if the co-multiplication $\psi$ satisfies the following:

(i) $\left(1_{G} * \psi\right) \psi=\left(\psi * 1_{G}\right) \psi$,

(ii) $\left(C * 1_{G}\right) \psi=1_{G}$ and $\left(1_{G} * C\right) \psi=1_{G}$, 
where $C: G \rightarrow Q$ is the homomorphism into the group of one element, and $G$ is identified with $G * Q$.

THEOREM C. [5] Let $X^{\prime}$ be a copy of $X$, and $x^{\prime} \in X^{\prime}$ will correspond to $x \in X$. Then the homomorphism $\psi: F(X) \rightarrow F(X) * F\left(X^{\prime}\right)$ such that $\psi(x)=$ $x \cdot x^{\prime} \in F(X) * F\left(X^{\prime}\right)$ is a co-multiplication for $F(X)$. This homomorphism is called the associated co-multiplication with $X$.

Let $X$ be a subset of the group $H$. Then $G_{n}(X)$ denotes all reduced words in $X$ whose length is not larger than $n$.

3. A category of p.f.b's. In this section we consider p.f.b.'s with their standard terminology [3], but not in the usual approach. In our study we do not keep the fiber fixed, but rather the base. Consequently the morphisms have to be changed. To keep our notation straight, for a given fixed topological space $X$, we define the objects and morphisms in the category.

Definition 1. [3] Let $A$ be a topological group acting effectively with continuous translation functions on the right of a topological space $S$. If the space of orbits of $S$ with the identification topology is homeomorphic to $X$, then the triple $\langle S, p, A\rangle$ is a p.f.b., where $p$ is the map sending $s \in S$ to $p(s) \in$ $X$, which corresponds under the homeomorphism to the orbit of $s$.

Definition 2. Let $\alpha_{i}=\left\langle S_{i}, p_{i}, A_{i}\right\rangle, i=1,2$, be p.f.b.'s. A morphism from $\alpha_{1}$ to $\alpha_{2}$ is a pair $\left\langle f, f_{S}\right\rangle$ where $f_{S}: S_{1} \rightarrow S_{2}$ is a map, and $f: A_{1} \rightarrow A_{2}$ is a homomorphism such that: (i) $f_{S}(s a)=f_{S}(s) \cdot f(a)$, (ii) $p_{2} f_{S}(s)=p_{1}(s)$.

Definition 3. Let $\alpha_{1}=\left\langle S_{1}, p_{1}, A_{1}\right\rangle$ be a locally trivial fiber bundle with a system of transition functions $\left\{g_{i j}\right\}$ relative to the open cover $\left\{U_{i}\right\}$, and let $f$ : $A_{1} \rightarrow A_{2}$ be a homomorphism into a topological group $A_{2}$. Then the p.f.b. $f_{*}\left(\alpha_{2}\right)=\left\langle S_{2}, p_{2}, A_{2}\right\rangle$ constructed by the system of transition functions $\left\{f, g_{i j}\right\}$ relative to $\left\{U_{i}\right\}$ is called the co-induced bundle of $\alpha_{1}$ by $f$. If $f_{S}: S_{1} \rightarrow S_{2}$ is the map induced by $f$, we get a morphism $\left\langle f, f_{S}\right\rangle: \alpha_{1} \rightarrow \alpha_{2}$.

Proposition 1. Let $\alpha_{1}$ and $\alpha_{2}$ be as in Definition 3. If $X, S_{1}, A_{1}, A_{2}$ are $k_{W}$ spaces so is $S_{2}$. (Proof in $\S 10$.)

We denote the category of locally trivial p.f.b.'s with morphisms as in Definition 2 by $F$.

4. The category of p.c.b.'s. We start this section with the definition of a p.c.b. Later on we will restrict our interest to a special kind of p.c.b.

Definition 4. The tuple $\langle T, \phi, A, F, \psi\rangle$ is called a p.c.b. with $T$ the total space, $\phi$ the co-operation, $A$ the co-base, $F$ the co-fiber if the following axioms hold: (i) $\langle F, \psi\rangle$ is a co-group. (ii) The continuous homomorphism $\phi$ makes the following diagram commutative: 


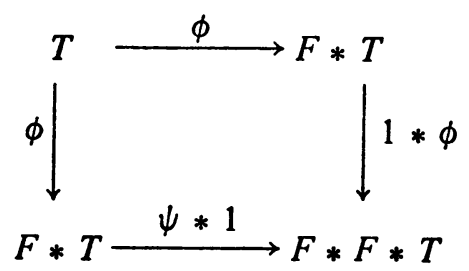

(iii) If $r_{1}$ and $r_{2}$ are the projections of $F * T$ on $F$ and $T$ respectively then $r_{2} \phi=1_{T}$ and $r_{1} \phi$ is an epimorphism. (iv) The group $A$ is imbedded by an isomorphism $i: A \rightarrow T$ onto the subgroup, $I=\{t \in T \mid \phi(t) \in T \subset F * T\}$ of the invariant elements of $T$ relative to $\phi$.

A morphism between the p.c.b.'s with the same co-group is defined next:

Definition 5. Let $\beta_{i}=\left\langle T_{i}, \phi_{i}, A_{i}, F, \psi\right\rangle, i=1,2$, be p.c.b.'s. Then $\left\langle f, f_{T}\right\rangle: \beta_{1} \rightarrow \beta_{2}$ is a morphism if the following diagram of groups and homomorphisms commutes:

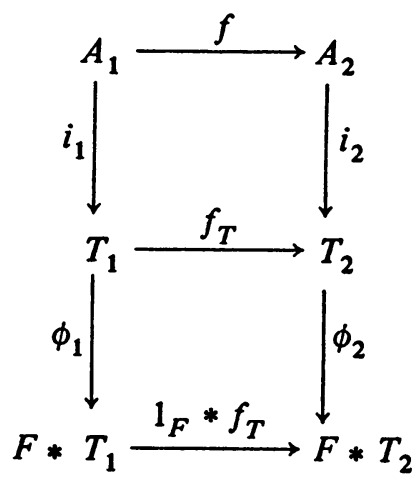

Proposition 2. Let $\beta_{1}=\left\langle T_{1}, \phi_{1}, A_{1}, F, \psi\right\rangle$ be a p.c.b., and let $f: A_{1} \rightarrow$ $A_{2}$ be a homomorphism into the topological group $A_{2}$. Then there exists $a$ p.c.b. $\beta_{2}=\left\langle T_{2}, \phi_{2}, A_{2}, F, \psi\right\rangle$, and a morphism $\left\langle f, f_{T}\right\rangle: \beta_{1} \rightarrow \beta_{2}$. The p.c.b. $\beta_{2}$ is called the p.c.b. induced from $\beta_{1}$ by $f$ and will be denoted by $f_{*}\left(\beta_{1}\right)$.

Proof. Let $T_{2}$ be the quotient group with the quotient topology of $T_{1} * A_{2}$ relative to the relations $a^{-1} \cdot f(a)=e$ for all $a \in A_{1}$. We denote the quotient homomorphism by $q$. The homomorphism $i_{2}$ is the restriction of $q$ to $A_{2}$, and the homomorphism $f_{T}$ is the restriction of $q$ to $T_{1}$. The co-operation $\phi_{2}$ is the unique homomorphism making the following diagram commutative:

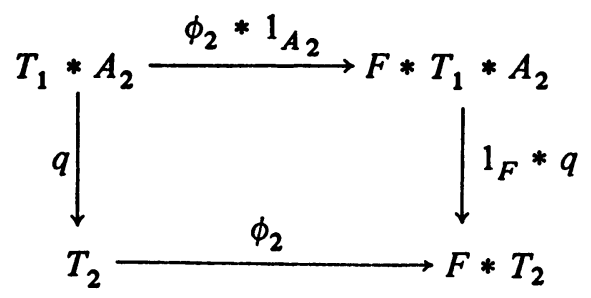


Proposition 3. Let $\beta_{1}$ and $\beta_{2}$ be as in Proposition 2. If $A_{1}, T_{1}, A_{2}$ are $k_{W}$ groups so is $T_{2}$. (The proof is in $\S 10$.)

We will be interested in p.c.b.'s with a fixed co-group of the following type: $(F(X), \psi)$, where $\psi$ is the co-multiplication associated with $X$. Of course for the existence of $F(X)$ we require $X$ to be completely regular from now on. Because of this restriction we can abbreviate the notation of a p.c.b. to $\langle T, \phi, A\rangle$.

Definition 6. $\langle T, \phi, A\rangle$ is called a locally trivial p.c.b. if the following holds: There exists a system $\left\{g_{\alpha}: U_{\alpha} \rightarrow T\right\}$ of continuous maps such that $\left\{U_{\alpha}\right\}$ is an open cover of $X$, and $r_{1} \phi g_{\alpha}=1_{U_{\alpha}}$ for all $U_{\alpha}$ of the cover $\left(r_{1}\right.$ : $F(X) * T \rightarrow F(X)$ the projection). The $\left\{g_{\alpha}\right\}$ are called the sections of $\langle T, \phi, A\rangle$.

Proposition 4. Let $\beta_{1}$ and $\beta_{2}$ be as in Proposition 2. Then if $\beta_{1}$ is a locally trivial p.c.b. so is $f_{*}\left(\beta_{1}\right)=\beta_{2}$.

Proof. If $\left\{g_{\alpha}\right\}$ are the sections of $\beta_{1}$ then $\left\{f_{T} g_{\alpha}\right\}$ are the sections of $\beta_{2}$. We denote the category of locally trivial p.c.b.'s by $C$.

5. The restriction functor $\delta$. In this section we reveal the technique by which we will study the structure of p.c.b.'s, via the well-known structure of associated p.f.b.'s.

Let $\beta_{i}=\left\langle T_{i}, \phi_{i}, A_{i}\right\rangle \in C, i=1,2$. Then, $T_{i S}=\phi^{-1}\left(X \times T_{i}\right)$, and $\phi_{i S}$ : $T_{i S} \rightarrow X$ is defined as follows: $\phi_{i S}=r_{1}\left(\left.\phi_{i}\right|_{T_{i S}}\right)$, with $r_{1}$ as in Definition 6 . If $\left\langle f, f_{T}\right\rangle: \beta_{1} \rightarrow \beta_{2}$ is a morphism in $C$, we denote by $f_{T S}: T_{1 S} \rightarrow T_{2 S}$ the map $f_{T} \mid T_{1 S}$. This construction makes sense because $X \times T_{i}$ is imbedded in $F(X) * T_{i}[8]$.

THEOREM 1. The function $\delta$ which assigns to each $\beta=\langle T, \phi, A\rangle \in \mathcal{C}$ the triple $\delta(\beta)=\left\langle T_{S}, \phi_{S}, A\right\rangle$ and to each morphism $\left\langle f, f_{T}\right\rangle: \beta_{1} \rightarrow \beta_{2} \in C$ the pair $\left\langle f, f_{T S}\right\rangle$ is a functor from $C$ to $F$. (The proof is straightforward.)

Proposition 5. Let $T$ and $X$ be $k_{W}$ spaces. Then so is $T_{S}$. (The proof is in $\S 10$.

6. The extension functor $\epsilon$. Let $\alpha=\langle S, p, A\rangle \in F$. We consider the following relations in $F(S)$ :

$$
\left\{s \cdot a \cdot(s a)^{-1}=e \mid s \in S, a \in A\right\} .
$$

Denote the quotient group with the quotient topology of $F(S)$ relative to the above relations by $S_{T}$, and the quotient homomorphism by $q: F(S) \rightarrow S_{T}$. We also denote by $p_{T}$ the unique homomorphism which makes the following diagram commutative: 


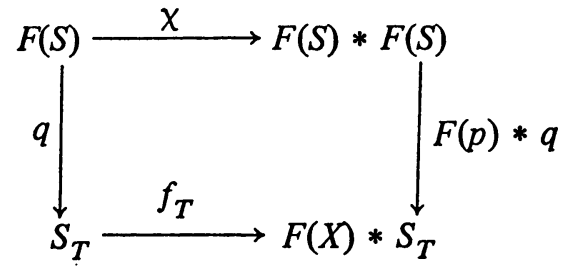

where $\chi$ is the homomorphism induced by the map which sends $s \in S$ to $s_{1} \cdot s_{2}$ $\in F(S) * F(S)$, where $s_{1}, s_{2}$ are copies of $s$ in the two components of the free product. For any $\left\langle f, f_{S}\right\rangle: \alpha_{1} \rightarrow \alpha_{2} \in F$ we define $f_{S T}: S_{1 T} \rightarrow S_{2 T}$, as the unique homomorphism which makes the following diagram commutative:

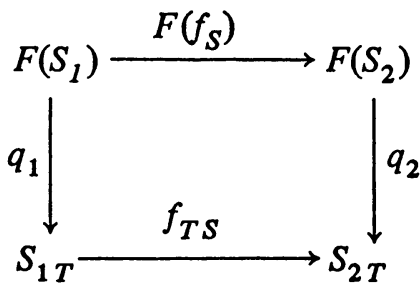

THEOREM 2. The function $\epsilon$ which assigns to each $\alpha=\langle s, p, A\rangle \in F$ the triple $\epsilon(\alpha)=\left\langle S_{T}, p_{T}, A\right\rangle$ and to each morphism $\left\langle f, f_{S}\right\rangle: \alpha_{1} \rightarrow \alpha_{2} \in F$ the morphism $\epsilon\left\langle f, f_{S}\right\rangle=\left\langle f, f_{S T}\right\rangle: \epsilon\left(\alpha_{1}\right) \rightarrow \epsilon\left(\alpha_{2}\right)$ is a functor from $F$ to $C$. (The proof is again straightforward.)

Proposition 6. If $S$ and $A$ of $\alpha$, in Theorem 2 , are $k_{W}$ spaces, so is $S_{T}$. (The proof is in $\S 10$.)

7. Relations between $\epsilon$ and $\delta$. We open this section with the remark that $\epsilon$ is a left adjoint functor to $\delta$. However for the classification theorem in the next section we need a stronger connection between these two functors. To achieve this we replace $C$ by its subcategory $e(F)$ which we denote by $C^{\prime}$. Under this condition we obtain the following result:

THEOREM 3. The functors $\epsilon$ and $\delta$ are inverses of each other.

Theorem 3 is the main tool which enables us to deduce properties of p.c.b.'s from p.f.b.'s.

Proposition 7. Let $\left\langle 1, f_{T}\right\rangle:\left\langle T_{1}, \phi_{1}, A\right\rangle \rightarrow\left\langle T_{2}, \phi_{2}, A\right\rangle \in C^{\prime}$. Then $\left\langle 1, f_{T}\right\rangle$ is an equivalence.

Proof. Since $\delta\left\langle 1, f_{T}\right\rangle$ is an equivalence in $F, \delta\left\langle 1, f_{T}\right\rangle$ is an equivalence in $C^{\prime}$

Proposition 8. Let $\left\langle f, f_{T}\right\rangle: \beta_{1} \rightarrow \beta_{2} \in C^{\prime}$. Then $f_{*}\left(\beta_{1}\right)$ is equivalent to $\beta_{2}$ 
Proof. This follows from the universal property of induced p.c.b.'s (i.e. that any morphism in $C^{\prime}$ factors through the canonical map into the induced p.c.b.) and Proposition 7.

Proposition 9. Let $f: A_{1} \rightarrow A_{2}$ be an homomorphism between two topological groups, and let $\beta_{1}=\left\langle T_{1}, \phi_{1}, A_{1}\right\rangle \in \mathcal{C}$. Then $f_{*}\left(\beta_{1}\right)$ and $\epsilon\left(f^{*}\left(\delta\left(\beta_{1}\right)\right)\right)$ are equivalent.

Proof. This is an easy consequence of Proposition 7.

At this point we would like to remark that we can replace $F$ by a subcategory $\mathrm{F}^{\prime}$ of all p.f.b.'s $\langle S, p, A\rangle$, such that $S$ and $A$ are $k_{W}$ spaces, and $C^{\prime}$ to be replaced by $C^{\prime \prime}$ whose objects $\langle T, \phi, A\rangle$ satisfy: $A, T$ are $k_{W}$ groups such that $T$ has the weak topology of $F_{n}\left(T_{S}\right)$. All results of this section hold for these categories with $\epsilon$ and $\delta$ properly changed.

We close this section with the notion of a trivial p.c.b.

Definition 7. The p.c.b. $\langle T, \phi, A\rangle$ is called trivial if $T=F(X) * A$ and $\phi=\psi * 1_{A}$.

Proposition 10. The functors $\epsilon$ and $\delta$ correspond trivial p.f.b.'s to trivial p.c.b.'s.

Proof. It is enough to show that $\delta$ maps a trivial p.c.b. to a trivial p.f.b. Since $F(X) \times A$ is embedded in $F(X) * A$, and $(F(X) * A)_{T}=X \times A$ the result follows.

8. The classification theorem for p.c.b.'s. In this section we present and prove a classification theorem for the category $C^{\prime \prime}$ of locally trivial p.c.b.'s. For the classification we use the functors $\delta$ and $\epsilon$, which reduce the problem to the category $F^{\prime}$. In this category we rely heavily on a result of Milnor's which is also extended in this section.

Let $X$ be a countable simplicial complex. Denote by $Z$ all finite $(n+1)$ tuples $\left(x_{n}, x_{n-1}, \ldots, x_{0}\right)$ of points in $X$ such that each pair $x_{i}, x_{i-1}$ lie in a common simplex of $X$. We denote by $\widetilde{Z}$ the equivalence classes of $Z$ under the relation: $\left(x_{n}, \ldots, x_{i+1}, x_{i}, x_{i-1}, x_{0}\right)$ is equivalent to $\left(x_{n}, \ldots, x_{i+1}, x_{i-1}, \ldots\right.$, $\left.x_{0}\right)$ whenever $x_{i}=x_{i-1}$, and denote the class of $\left(x_{n}, \ldots, x_{0}\right)$ by $\left[x_{n}, \ldots, x_{0}\right]$. We also use the following notation: (a) $\widetilde{E}(X)=\left\{\left[x_{n}, \ldots, e\right] \in \widetilde{Z}\right\} ;$ (b) $p: \widetilde{E}(X) \rightarrow$ $X$ such that $p\left(\left[x_{n}, \ldots, e\right]\right)=x_{n} ;($ c) $\widetilde{G}(X)=\operatorname{ker} \tilde{p}$.

THEOREM I [7]. $\langle\widetilde{E}(X), \widetilde{p}, \widetilde{G}(X)\rangle \equiv \xi \in F^{\prime}$.

ThEOREM II [7]. Every $\alpha \in F^{\prime}$ is co-induced from $\xi$.

The homomorphism which co-induces $\alpha$ of the theorem depends on a system of slicing functions of $\alpha$. We call this homomorphism a Milnor homomor- 
phism. Next we state the main result:

Theorem 4. Denote by $\eta=\epsilon(\xi)$; then: (a) Every p.c.b. $\alpha \in C^{\prime \prime}$ is induced by a Milnor homomorphism from $\eta$. (b) If $h_{0}, h_{1}: \widetilde{G}(X) \rightarrow A$ are loop homotopic homomorphisms (i.e. there exists a map $H: \widetilde{G}(X) \times[0,1] \rightarrow A$ such that $H(, t)$ for a fixed $t \in[0,1]$ is a homomorphism and $H(, 0)=h_{0}$, $\left.H(, 1)=h_{1}\right)$ then $h_{0} *(\eta)$ is equivalent to $h_{1} *(\eta)$. (c) If $k_{0}$ and $k_{1}$ are Milnor homomorphisms for two equivalent p.c.b. 's of $C^{\prime \prime}$, then they are loop homotopic.

The rest of this section is devoted to the proof of Theorem 4. As for part (a) it is an immediate consequence of Milnor's Theorem II and Proposition 9. The proof of (b) follows also from Proposition 9 and the following:

Proposition 11. Let $l_{0}, l_{1}: A \rightarrow A_{1}$ be two homomorphisms which are loop homotopic. If $\alpha=\langle S, p, A\rangle \in F^{\prime}$, then $l_{0}(\alpha)$ is equivalent to $l_{1} *(\alpha)$.

Proof. If $\left\{g_{i j}\right\}$ is a system of transition functions for $\alpha$ with the open cover $\left\{U_{\alpha}\right\}$ of $X$, then $\left\{h_{0} g_{i j}\right\}$ and $\left\{h_{1} g_{i j}\right\}$ are systems of transition functions with the same open cover for $l_{0 *}(\alpha)$ and $l_{1 *}(\alpha)$ respectively. Let $H$ be the loop homotopy given in the theorem, then $H\left(g_{i j} \times 1_{I}\right)$ is a system of transition functions for the open cover $\left\{U_{\alpha} \times I\right\}$ of $X \times I$. By Theorem 9.8, p. 51 of [3] we conclude that $l_{0 *}(\alpha)$ and $l_{1} *(\alpha)$ are equivalent.

The proof of part (c) of Theorem 4 is again a result of Proposition 9 and the following:

Proposition 12. Let $k_{0}, k_{1}: \widetilde{G}(X) \rightarrow A$ be Milnor homomorphisms for equivalent p.f.b.'s in $F^{\prime}$. Then they are loop homotopic.

The proof of this is done in several stages:

LEMMA 1. Theorem I stated above is correct if we replace the simplicial complex $X$ by the complex $X \times I$, whose cells are of the form $\tau \times I$, with $\tau$ a simplex of $X$.

The proof is the same that Milnor gives for $X$ in [7].

LEMMA 2. Theorem II holds if we replace $X$ by $X \times I$ as in Lemma 1 .

Proof. This is again, word for word, the proof of Theorem 5.1 in [7] with the following observation: The proof of Proposition 5.3 which provides a system of slicing functions, depends on the fact that the equivariant cohomology groups $H^{k}\left(U \bmod \Delta, B_{n}, T\right)$ are all trivial, since simplexes are convex. This fact is also true in our case because $\tau \times I$ are also convex.

Lemma 3. Let $\langle\bar{S}, \bar{p}, A\rangle$ be a locally trivial p.f.b. over $X \times I$. Let $\left.\bar{S}\right|_{X \times\{0\}}$ and $\left.\bar{S}\right|_{X \times\{1\}}$ have two systems of slicing functions. Then $\langle\bar{S}, \bar{p}, A\rangle$ has a system of slicing functions which extend the two already given. 
PROOF. The proof is again similar to the proof of 5.3 of [7], where this time we use the following homotopy: for $(x, r) \times(y, s) \in(\tau \times I) \times(\pi \times I)$

$$
\begin{aligned}
& H((x, r),(y s), t) \\
& =\left\{\begin{array}{l}
(x, r+t(s-r)),(y, s+t(r-s)), \quad 0 \leqslant t \leqslant \frac{1}{2}, \\
\left(x+\left(t-\frac{1}{2}\right)(y-x), \frac{r+s}{2}+\left(\frac{r+s}{2}-\frac{1}{2}\right)\left(t-\frac{1}{2}\right)(|x-y|)\right), \\
\left(y+\left(t-\frac{1}{2}\right)(x-y), \frac{r+s}{2}+\left(\frac{r+s}{2}-\frac{1}{2}\right)\left(t-\frac{1}{2}(|x-y|)\right),\right. \\
\frac{1}{2} \leqslant t \leqslant 1,
\end{array}\right.
\end{aligned}
$$

such that if for some $(x, r),(y, s)$ there exists a $t_{0} \geqslant 1 / 2$ such that $H\left((x, r),(y, s), t_{0}\right)$ is in the diagonal of $X \times I$ or in $(X \times\{0\}) \times(X \times\{0\})$ or in $(X \times\{1\}) \times(X \times\{1\})$ then $H((x, r),(y, s), t)=H\left((x, r),(y, s), \mathrm{t}_{0}\right)$ for all $t \geqslant t_{0}$.

Proof of Proposition 12. Since $k_{0^{*}}(\xi)$ is equivalent to $k_{1} *(\xi)$, there are two homotopic maps $f_{0}, f_{1}: X \rightarrow B_{A}$ (where $B_{A}$ is the classifying space for fiber bundles with fiber $A$ ). Let $H$ be the homotopy from $f_{0}$ to $f_{1}$ and $\langle\bar{S}, \bar{p}, A\rangle$ the pull back bundle of the universal bundle $\left(\widetilde{E}_{A}, \tilde{p}, B_{A}\right)$ via $H$. By Lemma 3 this bundle has a system of slicing functions extending the original two given. By Lemma 2 the p.f.b. $\langle\bar{S}, \bar{p}, A\rangle$ is induced by a homomorphism $\widetilde{H}: \widetilde{G}(X \times I) \rightarrow A$. Furthermore there exists a homomorphism $J: \widetilde{G}(X) \times I$ $\rightarrow \widetilde{G}(X \times I)$ defined as follows: the pair $(\alpha, t)$ is mapped to the tuple which starts on the right with $(e, 0)$, the next coordinates are those of $\alpha$ embedded in $X \times t$, and the last one is again $(e, 0)$. Combining the last two maps we get a loop homotopy $H=\widetilde{H} J: \widetilde{G}(X) \times I \rightarrow A$ such that $H \mid \widetilde{G}(X) \times\{0\}=k_{0}$, and $H \mid \widetilde{G}(X) \times\{1\}$ is conjugate to $k_{1}$. However from the proof of Theorem II it is apparent that the conjugating element is in the path component of the identity of $A$. Thus $H \mid \widetilde{G}(X) \times\{1\}$ is loop homotopic to $k_{1}$ and we conclude that $k_{0}$ and $k_{1}$ are loop homotopic.

9. An example. In this section we present a p.c.b. which arises in quite a natural way, and show in fact that many p.c.b.'s are of this form. This is done by relating p.c.b.'s to principal co-fibrations.

Let $X$ be a connected subcomplex of a countable connected simplicial complex $Y$. Let $e \in X \subset Y$ be the base point. We denote by $C X$ the unreduced cone over $X$, i.e. $C X=X \times I / X \times\{1\}$ and by $\Sigma X$ the unreduced suspension of $X$, i.e. $\Sigma X=X \times I / X \times(\{0\},\{1\})$. We mark the point resulting from the collapsing of $X \times\{0\}$ by $v_{0}$ and of $X \times\{1\}$ by $v_{1}$. Let $Z$ be the identification 
space of $Y \cup C X$ where $(x, 0) \in C X$ is identified with $x \in Y . C X, \Sigma X$ and $Z$ are again countable connected simplicial complexes.

Let $\hat{E}(Z)$ be a subcomplex of $\widetilde{E}(Z)$ (see $\S 8$ ) consisting of all tuples such that no coordinate lies in $\left(Z-\left(C X-v_{1}\right)\right)$ unless it is the first on the left. Let $\hat{G}(Z)$ denote those tuples of $\hat{E}(Z)$, with first coordinate from the left equal to $e$. Every element $a$ of $\hat{G}(Z)$ can be represented by a product of special elements $a=a_{1} \cdot a_{2} \cdots a_{n}$, with $a_{i} \in \hat{G}(Z)$, where $a_{i}$ is one of the following types: $\{[w] \in \widetilde{G}(Y)\},\left\{\left[u \cdot x \cdot v_{1} \cdot e\right] \mid u\right.$ is a tuple in $Y$ that starts on the left with $e$, and $x \in X\}, \quad\left\{\left[e \cdot v_{1} \cdot x \cdot u\right] \mid u \in \widetilde{E}[Y], x \in X\right\},\left\{\left[e \cdot v_{1} \cdot x_{1} \cdot u \cdot x_{2} \cdot v_{1} \cdot e\right] \mid\right.$ $u$ is a tuple in $Y$ \}. Define a homomorphism $\hat{\phi}: \hat{G}(Z) \rightarrow F(X) * \hat{G}(Z)$ such that:

$\hat{\phi}(a)= \begin{cases}{[w]} & \text { for } a=[w], \\ x \cdot\left[e \cdot v_{1} \cdot x \cdot u\right] & \text { for } a=\left[e \cdot v_{1} \cdot x \cdot u\right], \\ {\left[u \cdot x \cdot v_{1} \cdot e\right] \cdot x^{-1}} & \text { for } a=\left[u \cdot x \cdot v_{1} \cdot e\right], \\ x_{1}\left[e \cdot v_{1} \cdot x_{1} \cdot u \cdot x_{2} \cdot v_{1} \cdot e\right] x_{2}^{-1} & \text { for } a=\left[e \cdot v_{1} \cdot x_{1} \cdot u \cdot x_{2} \cdot v_{1} \cdot e\right]\end{cases}$

It is easy to check that $\hat{\phi}$ is well defined.

Theorem 5. $\langle\hat{G}(Z), \hat{\phi}, \widetilde{G}(Y), F(X)\rangle$ is a locally trivial p.c.b. in $C^{\prime \prime}$ (see §7).

Proof. Let $S=\{a \in \hat{G}(Z) \mid \hat{\phi}(a) \in X \times \hat{G}(Z)\}$. These are all elements of the form $\left\{\left[e \cdot v_{1} \cdot x \cdot u\right] \mid u \in \widetilde{E}(Y)\right\}$ which are homeomorphic to $\{a \in \widetilde{E}(Y)$ whose first element on the left is in $X\}$. We denote this set by $\widetilde{E}(Y, X)$. Since $\left\langle S=\widetilde{E}(Y, X), \phi_{S}, \widetilde{G}(Y)\right\rangle$ is the pull back p.f.b. of the p.f.b. $\langle\widetilde{E}(Y), \widetilde{P}, \widetilde{G}(Y)\rangle$ via the inclusion map of $X$ into $Y$, the pull back bundle is also locally trivial. The result now follows from Theorem 2 .

To see the generality of the above example consider the following: Let $f: X \rightarrow Y$ be any map between spaces of the homotopy type of countable c.w. complexes since up to homotopy type $f$ can be replaced by the inclusion of a countable simplicial complex into another countable simplicial complex. We consider this situation, $i: X \rightarrow Y$. Let $Y \rightarrow Y \cup_{i} C X \rightarrow \Sigma X$ be the principal cofibration induced by $i$ from the co-fibration $X \rightarrow C X \rightarrow \Sigma X$.

THEOREM 6. The total space, the co-base, and the co-fiber, of the p.c.b. of Theorem 5, are of the homotopy type of the loops of: the total space, the co-base and the co-fiber of the principal co-fibration $Y \rightarrow Y \cup_{i} C X \rightarrow \Sigma X$.

Proof. That $\widetilde{G}(Y)$ has the homotopy type of $\Omega Y$ follows from [7]. The rest of the proof is split up in the next two propositions:

Proposition 13. $\hat{G}(Z)$ has the homotopy type of $\widetilde{G}(Z)$, thus also of $\Omega Z$. 
Proposition 14. $F(X)$ has the homotopy of $\Omega \Sigma X$.

Proof of Proposition 13. We start with the proof that $\langle\hat{E}(Z), \hat{P}, \hat{G}(Z)\rangle$ is a p.f.b. of $F^{\prime \prime}$, where $\hat{P}$ : $\hat{E}(Z) \rightarrow Z$ sends a tuple of $\hat{E}(Z)$ to its first coordinate on the right. It is clear that $\hat{G}(Z)$ operates effectively on $\hat{E}(Z)$. The rest follows by the use of Milnor's arguments in [7] for proving that $\langle\widetilde{E}(X), \widetilde{P}, \widetilde{G}(X)\rangle$ is a universal p.f.b. with the following changes. For the coordinate neighborhood of $v_{1}$ there is no change. For the coordinate neighborhoods st $(u)$ where $u$ is a vertex of $X$, the connection of $u$ with a point in $s t(u) \cap X$ is as in the original proof, while the connection with a point $t \in s t(u) \cap(C X-X)$ is via an extra coordinate in $X$, which lies in a straight line under the point in $C X-X$, between $t$ and $u$. The rest of the proof is with no change. The rest of the proof follows by the five lemma and the homotopy ladder of the following commutative diagram of p.f.b.'s

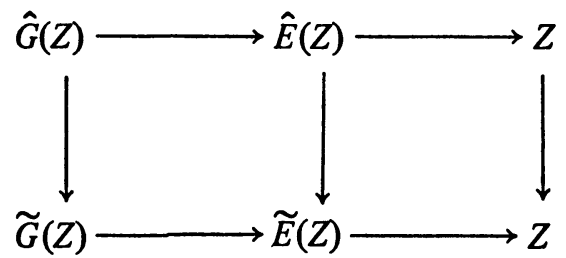

where the vertical maps are inclusions.

Proof of Proposition 14. Let $\bar{G}(\Sigma X)$ be the subgroup of $\widetilde{G}(\Sigma X)$ generated by elements of the form $\left[e \cdot v_{1} \cdot x \cdot v_{0} \cdot e\right] . \bar{G}(\Sigma X)$ is homeomorphic to $F(X)$ via the map which sends $x$ to $\left[e \cdot v_{1} \cdot x \cdot v_{2} \cdot e\right]$. To complete the proof it suffices to show that $\bar{G}(\Sigma X)$ is of the homotopy type of $\widetilde{G}(\Sigma X)$. Define $\bar{E}(\Sigma X) \subset \widetilde{E}(\Sigma X)$ which are those of $\bar{G}(\Sigma X)$ with the exception that they do not have to end in $e$ but anywhere in $\Sigma X$. If $\bar{P}: \bar{E}(X) \rightarrow X$ is the projection on the first coordinate from the left then as in the proof of Proposition 13 we get that $\langle\bar{E}(X), \bar{P}, \bar{G}(X)\rangle$ is a p.f.b. The rest of the proof is similar to the proof of Proposition 13, this time with the following diagram:

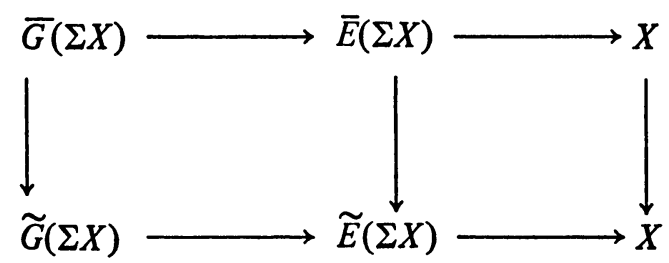

where the vertical maps are inclusions.

10. Proofs.

Proof of Proposition 1. The total space $S_{2}$ is homeomorphic to the 
total space of the fiber bundle $\alpha_{1}\left[A_{2}\right][3, \mathrm{p} .44]$, which is a quotient space of the $k_{W}$ space $S_{1} \times A_{2}$.

Proof of Proposition 3. The only fact not proved in Proposition 2 is that $T_{2}$ is Hausdorff. This follows from Propositions 1 and 9.

Proof of Proposition 5. Claim $F(X) \times T$ is closed in $F(X) * T$. Since $X$ is closed in $F(X)$ [1], $X \times T$ is closed in $F(X) * T$. Thus $T_{S}$ being the inverse image of a closed set is closed and therefore also $k_{W}$. Next we prove the claim. Let $F(X)=\bigcup X_{i}$ and $T=\bigcup Y_{i}$ be $k_{W}$ decompositions, then $F(X) * T$ $=\bigcup h\left(\left(X_{i} \vee Y_{i}\right)^{i}\right)$ is also a $k_{W}$ decomposition, where $h$ sends a word to its reduced form. If we denote $X_{n}$ and $Y_{n}$ by $K_{n}^{0}$ and $K_{n}^{1}$ respectively then $h\left(\left(X_{n} \vee Y_{n}\right)^{n}\right)$ can be replaced by a finite union of the following compact sets: $h\left(\Pi_{j=1}^{n} K_{n}^{i_{j}}\right)$ where $i_{j}$ takes values 0 and 1 . Now it is clear that

$$
F(X) \times T \cap h\left(\left(X_{n} \vee Y_{n}\right)^{n}\right)=\bigcup_{\substack{k+l=n \\ k, l \geqslant 0}} h\left(\left(X_{n}\right)^{k}\right) \times h\left(\left(T_{n}\right)^{l}\right)
$$

Since the right hand side of the last equality is compact the claim follows.

(Note that the claim and the proof generalize trivially to a finite number of groups.)

Proof of Proposition 6. Let $S=\bigcup_{k=1}^{\infty} S_{k}$ be a $k_{w}$ decomposition. Define the following sets:

$$
A_{k}=\tau\left(S_{k} \times S_{k}\right), \quad A_{k}^{\prime}=A_{k} \cup\left(A_{k}^{-1}\right), \quad S_{k}^{\prime}=\mu^{\prime}\left(S_{k} \times A_{k}^{\prime} \times \cdots \times A_{k}^{\prime}\right),
$$

where $\tau$ is the translation function of $\langle S, P, A\rangle$ (Definition 1), $\mu^{\prime}$ is defined on $S_{k} \times\left(A_{k}^{\prime}\right)^{k}$ and is induced by $\mu$, the action of $A$ on $S$, and $\left(A_{k}^{-1}\right)$ are the inverses of $A_{k}$ in the group $A$. We also use the following notation: $T_{k}=\left\{s \cdot a \cdot(s a)^{-1},(s a)^{-1} \cdot s \cdot a, s \cdot a^{-1} \cdot\left(s\left(a^{-1}\right)\right)^{-1},\left(s\left(a^{-1}\right)\right)^{-1} \cdot s \cdot a^{-1} \mid s \in\right.$ $\left.S_{k}^{\prime}, a \in A_{k}^{\prime}\right\}, H_{k}^{\prime}=\left\{u^{-1} \cdot v \cdot u \mid u \in F_{k}\left(S_{k}^{\prime}\right), v \in T_{k}^{ \pm 1}\right\}, H^{\prime}=\bigcup_{k=1}^{\infty} H_{k}^{\prime}, H_{k}^{\prime \prime}=$ $F_{k}\left(H_{k}^{\prime}\right), H^{\prime \prime}=\bigcup_{k=1}^{\infty} H_{k}^{\prime \prime}, H_{k}=h\left(H_{k}^{\prime \prime}\right)$ and $H=\bigcup_{k=1}^{\infty} H_{k}$, where $h$ sends a word to its reduced form in elements of $S$. It is obvious that $H=\operatorname{ker} q$, and that each $H_{k}$ is compact. Our problem is now reduced to showing that $H \cap F_{k}\left(S_{k}\right) \subset H_{k}$. This is the outcome of the following claims which actually amount to the fact that an element in $H$, not in $H_{k}$, cannot be in $F_{k}\left(S_{k}\right)$.

For a word $w$ in $F(S)$ we denote by $|W|$ its length in elements of $S$. We say that the words $e_{1}, e_{2} \in F(S)$ have a link of the first, second, third or fourth kind if one of the following holds respectively: $\left|e_{1} \cdot e_{2}\right|>\max \left\{\left|e_{1}\right|,\left|e_{2}\right|\right\}$, $\left|e_{1} \cdot e_{2}\right|=\max \left\{\left|e_{1}\right|,\left|e_{2}\right|\right\},\left|e_{1} \cdot e_{2}\right|=\max \left\{\left|e_{1}\right|,\left|e_{2}\right|\right\}-1,\left|e_{1} \cdot e_{2}\right|<$ $\max \left\{\left|e_{1}\right|,\left|e_{2}\right|\right\}-1$. Let $W=c_{1} \cdot c_{2} \cdots c_{k}$ be a representation in elements of $H^{\prime}$ of a word in $F(S)$, such that $c_{i}$ cannot be represented by $c_{i}=d_{i}^{1} \cdots d_{i}^{i}, d_{i}^{j} \in$ $H^{\prime}$, with $\max _{1 \leqslant j \leqslant l}\left|d_{i}^{j}\right|<\left|c_{i}\right|$ for all $1 \leqslant i \leqslant k$. Such a representation will be 
called proper. Every word of $F(S)$ has a proper representation.

Claim 1. Let $w=c_{1} \cdot c_{2} \cdots c_{k}$ be a proper representation. Then between any two pairs of consecutive elements of $\left\{c_{i}\right\}$ with links of the third kind, there must be a pair of consecutive elements, with link of the first kind. There is no link of the fourth kind.

This claim is achieved by observing that in each of the many possibilities, if the claim is not satisfied, the representation cannot be proper. As an easy consequence we get:

Claim 2. If $w=c_{1} \cdots c_{k}$ is a proper representation, then $|w| \geqslant\left[\left|c_{i}\right| / 2\right]$ for $1 \leqslant i \leqslant k$, and $|w| \geqslant k$. $([x]=$ the largest integer $\leqslant x$. $)$

Claim 3. If $w \in F\left(S_{k}\right) \cap H$, then $w$ has a representation in elements of $F\left(S_{k}^{\prime}\right) \cap H^{\prime}$. This follows from the definitions of $\left\{S_{k}^{\prime}\right\},\left\{A_{k}^{\prime}\right\}$ and the operations needed in the proof of Claim 2.

The proof of Proposition 6 follows now at once.

\section{REFERENCES}

1. M. I. Graev, Free topological groups, Izv. Akad. Nauk SSSR Ser. Mat. 12 (1948), 279-324; English transl., Amer. Math. Soc. Transl. (1) 8 (1962), 305-364. MR 10, 11.

2. - On free products of topological groups, Izv. Akad. Nauk SSSR Ser. Mat. 14 (1950), 343-354. (Russian) MR 12, 158.

3. D. H. Husemoller, Fibre bundles, McGraw-Hill, New York, 1966. MR 37 \#821.

4. P. J. Hilton, Homotopy and duality, Lecture Notes, Cornell Univ., Ithaca, N. Y., 1959.

5. E. Katz, Topological groups with co-monoid structures.

6. J. Mack, S. A. Morris and E. T. Ordman, Free topological groups and the projec. tive dimension of a locally compact abelian group, Proc. Amer. Math. Soc. 40 (1973), 303308. MR 47\#8755.

7. J. W. Milnor, Constructions of universal bundles. I, Ann. of Math. (2) 63 (1956), 272-284. MR 17, 994.

8. E. T. Ordman, Free products of topological groups which are $k_{\omega}$-spaces, Trans. Amer. Math. Soc. 191 (1974), 61-73.

9. N. H. Schlomiuk, Principal cofibrations in the category of simplicial groups, Trans. Amer. Math. Soc. 146 (1969), 151-165. MR 41 \#2686.

DEPARTMENT OF MATHEMATICAL SCIENCES, TEL-AVIV UNIVERSITY, TELAVIV, ISRAEL

Current address: Department of Mathematics, Haifa University, Haifa, Israel 